\title{
Induced Circuits in Graphs on Surfaces
}

\author{
ALEXANDER SCHRIJVER
}

\begin{abstract}
We show that for any fixed surface $S$ there exists a polynomial-time algorithm to test if there exists an induced circuit traversing two given vertices $r$ and $s$ of an undirected graph $G$ embedded on $S$. (An induced circuit is a circuit without chords.) The general problem (not fixing $S$ ) is NP-complete. In fact, for each fixed surface $S$ there exists a polynomial-time to find a maximum number of $r-s$ paths in $G$ such that any two form an induced circuit.
\end{abstract}

\section{Introduction}

In this paper we show that the following problem is solvable in polynomial time, for any fixed compact surface $S$ :

$$
\begin{aligned}
& \text { given: an undirected graph } G=(V, E) \text { embedded on } S \text { and } \\
& \text { two vertices } r \text { and } s \text { of } G \text {; } \\
& \text { find: an induced circuit in } G \text { that traverses } r \text { and } s .
\end{aligned}
$$

An induced circuit is a circuit having no chords. The problem is NP-complete for general undirected graphs, as was shown by Bienstock [1]. In [2] the problem was shown to be solvable in polynomial time for planar graphs. In fact we show that for any fixed compact surface $S$ the problem:

$$
\begin{aligned}
& \text { given: an undirected graph } G=(V, E) \text { embedded on } S \text { and } \\
& \text { two vertices } r \text { and } s \text { of } G ; \\
& \text { find: a maximum number of } r-s \text { paths in } G \text { any two of } \\
& \text { which form an induced circuit; }
\end{aligned}
$$

is solvable in polynomial time.

Our method uses a variant of a method developed in [3] to derive, for any fixed $k$, a polynomial-time algorithm for the $k$ disjoint paths problem in directed

\footnotetext{
${ }^{0} 1991$ Mathematics Subject Classification: Primary 05C10, 05C38, 05C85, Secondary 68Q25, 68R10

This paper is in final form and no version of it will be submitted for publication elsewhere.
}

(C) 1993 American Mathematical Society $0271-4132 / 93 \$ 1.00+\$ .25$ per page 
planar graphs. (This problem is NP-complete for general directed graphs, even for $k=2$.) The present method is based on cohomology over free boolean groups.

\section{Free boolean groups}

The free boolean group $B_{k}$ is the group generated by $g_{1}, g_{2}, \ldots, g_{k}$, with relations $g_{j}^{2}=1$ for $j=1, \ldots, k$. So $B_{k}$ consists of all words $b_{1} b_{2} \ldots b_{t}$ where $t \geq 0$ and $b_{1}, \ldots, b_{t} \in\left\{g_{1}, \ldots, g_{k}\right\}$ such that $b_{i} \neq b_{i-1}$ for $i=2, \ldots t$. The product $x \cdot y$ of two such words is obtained from the concatenation $x y$ by deleting iteratively all occurrences of any pair $g_{j} g_{j}$. This defines a group, with unit element 1 equal to the empty word $\emptyset$.

We call $g_{1}, \ldots, g_{k}$ generators or symbols. Note that

$$
B_{1} \subset B_{2} \subset B_{3} \subset \cdots \text {. }
$$

The size $|x|$ of a word $x$ is the number of symbols occurring in it, counting multiplicities. A word $y$ is called a segment of word $w$ if $w=x y z$ for certain words $x, z$. If $w=y z$ for some word $z, y$ is called a beginning segment of $w$, denoted by $y \leq w$. This partial order gives trivially a lattice if we extend $B_{k}$ with an element $\infty$ at infinity. Denote the meet and join by $\wedge$ and $\vee$.

We prove two useful lemmas.

Lemma 1. For all $x, y, z \in B_{k}$ one has:

$$
\begin{aligned}
& x \leq y \cdot z \text { and } z \leq y^{-1} \cdot x \Longleftrightarrow x^{-1} \cdot y \cdot z=1 \text { or } y=x w z^{-1} \\
& \text { for some word } w \text {. }
\end{aligned}
$$

Proof. $\Longleftarrow$ being easy, we show $\Longrightarrow$. Let $w:=x^{-1} \cdot y \cdot z$. As $x \leq y \cdot z, y \cdot z=x w$; and as $z \leq y^{-1} \cdot x, y^{-1} \cdot x=z w^{-1}$, that is, $x^{-1} \cdot y=w z^{-1}$. Hence if $w \neq 1$ then $x w z^{-1}=x \cdot w \cdot z^{-1}=y$.

Lemma 2. Let $x, y \in B_{k}$. If $x \not \leq y$ then the first symbol of $x^{-1}$ is equal to the first symbol of $x^{-1} \cdot y$.

Proof. Let $z:=x \wedge y$. So $x^{-1} \cdot y$ is the concatenation of $x^{-1} \cdot z$ and $z^{-1} \cdot y$. Since $x^{-1} z \neq 1$, the first symbol of $x^{-1} \cdot y$ is equal to the first symbol of $x^{-1} \cdot z$. Since $x^{-1} z \neq 1$ and $z \leq x$, the first symbol of $x^{-1} \cdot z$ is equal to the first symbol of $x^{-1}$. Hence the first symbol of $x^{-1}$ is equal to the first symbol of $x^{-1} \cdot y$.

\section{The cohomology feasibility problem for free boolean groups}

Let $D=(V, A)$ be a weakly connected directed graph, let $r \in V$, and let $(G, \cdot)$ be a group. Two functions $\phi, \psi: A \longrightarrow G$ are called $r$-cohomologous if there exists a function $f: V \longrightarrow G$ such that

$$
\begin{aligned}
& \text { (i) } f(r)=1 \\
& \text { (ii) } \psi(a)=f(u)^{-1} \cdot \phi(a) \cdot f(w) \text { for each } \operatorname{arc} a=(u, w) .
\end{aligned}
$$


This clearly gives an equivalence relation.

Consider the following cohomology feasibility problem (for free boolean groups):

$$
\begin{aligned}
\text { given: } & \text { a weakly connected directed graph } D=(V, A) \text {, a } \\
& \text { vertex } r \text {, and a function } \phi: A \longrightarrow B_{k} ; \\
\text { find: } & \text { a function } \psi: A \longrightarrow B_{k} \text { such that } \psi \text { is } r \text { - } \\
& \text { cohomologous to } \phi \text { and such that }|\psi(a)| \leq 1 \text { for each } \\
& \text { arc } a \text { (if there is one). }
\end{aligned}
$$

We give a polynomial-time algorithm for this problem. The running time of the algorithm is bounded by a polynomial in $|A|+\sigma+k$, where $\sigma$ is the maximum size of the words $\phi(a)$ (without loss of generality, $\sigma \geq 1$ ).

We may assume that with each arc $a=(u, w)$ also $a^{-1}:=(w, u)$ is an arc of $D$, with $\phi\left(a^{-1}\right)=\phi(a)^{-1}$.

Note that, by the definition of $r$-cohomologous, equivalent to finding a $\psi$ as in (6), is finding a function $f: V \longrightarrow B_{k}$ satisfying:

$$
\begin{aligned}
& \text { (i) } f(r)=1 \text {; } \\
& \text { (ii) for each arc } a=(u, w):\left|f(u)^{-1} \cdot \phi(a) \cdot f(w)\right| \leq 1 \text {. }
\end{aligned}
$$

We call such a function $f$ feasible.

It turns out to be useful to introduce the concept of 'pre-feasible' function. A function $f: V \longrightarrow B_{k}$ is pre-feasible if

(i) $f(r)=1$;

(ii) for each arc $a=(u, w)$ : if $\left|f(u)^{-1} \cdot \phi(a) \cdot f(w)\right|>1$ then $\phi(a)=f(u) y f(w)^{-1}$ for some word $y$.

Pre-feasibility behaves nicely with respect to the partial order $\leq$ on the set $B_{k}^{V}$ of all functions $f: V \longrightarrow B_{k}$ induced by the partial order $\leq$ on $B_{k}$ as: $f \leq g \Leftrightarrow f(v) \leq g(v)$ for each $v \in V$. It is easy to see that $B_{k}^{V}$ forms a lattice if we add an element $\infty$ at infinity. Let $\wedge$ and $\vee$ denote the meet and join. Then:

Proposition 1. If $f_{1}$ and $f_{2}$ are pre-feasible, then so is $f:=f_{1} \wedge f_{2}$.

Proof. Clearly $f(r)=1$. Suppose $\left|f(u)^{-1} \cdot \phi(a) \cdot f(w)\right|>1$ for some arc $a=(u, w)$. We show $\phi(a)=f(u) y f(w)^{-1}$ for some $y$. By (4) we may assume by symmetry that $f(u) \not \subset \phi(a) \cdot f(w)$. Since $f(w)=f_{1}(w) \wedge f_{2}(w)$, there is an $i \in\{1,2\}$ such that $f(u)^{-1} \cdot \phi(a) \cdot f_{i}(w)$ contains $f(u)^{-1} \cdot \phi(a) \cdot f(w)$ as a beginning segment. Without loss of generality, $i=1$. So $\left|f(u)^{-1} \cdot \phi(a) \cdot f_{1}(w)\right|>$ 1. As $f(u) \not \subset \phi(a) \cdot f(w)$, by Lemma 2 , the first symbols of $f(u)^{-1}$ and $f(u)^{-1} \cdot \phi(a) \cdot f(w)$ are equal. Since $f(u)^{-1} \cdot \phi(a) \cdot f(w) \leq f(u)^{-1} \cdot \phi(a) \cdot f_{1}(w)$, it follows that the first symbols of $f(u)^{-1}$ and $f(u)^{-1} \cdot \phi(a) \cdot f_{1}(w)$ are equal. So $f_{1}(u)^{-1} \cdot \phi(a) \cdot f_{1}(w)$ contains $f(u)^{-1} \cdot \phi(a) \cdot f_{1}(w)$ as segment. Hence $\left|f_{1}(u)^{-1} \cdot \phi(a) \cdot f_{1}(w)\right|>1$. As $f_{1}$ is pre-feasible, $\phi(a)=f_{1}(u) y^{\prime} f_{1}(w)^{-1}$ for some $y^{\prime}$. Since $f(u) \leq f_{1}(u)$ and $f(w) \leq f_{1}(w)$ this implies $\phi(a)=f(u) y f(w)^{-1}$ for some $y$. 
So for any function $f: V \longrightarrow B_{k}$ there exists a unique smallest pre-feasible function $\bar{f} \geq f$, provided there exists at least one pre-feasible function $g \geq f$. If no such $g$ exists we set $\bar{f}:=\infty$. In the next section we show that $\bar{f}$ can be found in polynomial time for any given $f$.

We first note:

Proposition 2. If $\bar{f}$ is finite then

$$
\begin{aligned}
& \text { (i) } f(r)=1 \\
& \text { (ii) }|f(v)|<(\sigma+1)|V| \text { for each vertex } v \text {; } \\
& \text { (iii) } f(u) \leq \phi(a) \cdot f(w) \text { or } f(w) \leq \phi(a)^{-1} \cdot f(u) \text { for each } \\
& \text { arc } a=(u, w) \text { with }\left|f(u)^{-1} \cdot \phi(a) \cdot f(w)\right|>1 \text {. }
\end{aligned}
$$

Proof. Let $\bar{f}$ be finite. Trivially $f(r) \leq \bar{f}(r)=1$. Moreover, let $a_{1}, \ldots, a_{t}$ form a simple path from $r$ to $v$. By induction on $t$ one shows $|\bar{f}(v)| \leq(\sigma+1) t$. (Indeed, let $a_{t}=(u, v)$. If $\left|\bar{f}(u)^{-1} \cdot \phi(a) \cdot \bar{f}(v)\right| \leq 1$ then by induction $|\bar{f}(u)| \leq(\sigma+1)(t-1)$, and hence $|\bar{f}(v)| \leq \bar{f}(u)|+| \phi(a) \mid+1 \leq(\sigma+1) t$. If $\left|\bar{f}(u)^{-1} \cdot \phi(a) \cdot \bar{f}(v)\right|>1$ then by $(8) \bar{f}(v)$ is a segment of $\phi(a)$ and hence $|\bar{f}(v)| \leq \sigma \leq(\sigma+1) t$.) So $|f(v)| \leq|\bar{f}(v)|<(\sigma+1)|V|$.

To see (iii), assume that $f(u) \not \leq \phi(a) \cdot f(w)$ and $f(w) \not \leq \phi\left(a^{-1}\right) \cdot f(u)$. So by Lemma 2 the first symbol of $f(u)^{-1} \cdot \phi(a) \cdot f(w)$ is equal to the first symbol of $f(u)^{-1}$. Similarly, the last symbol of $f(u)^{-1} \cdot \phi(a) \cdot f(w)$ is equal to the last symbol of $f(w)$. Since $f(u) \leq \bar{f}(u)$ and $f(w) \leq \bar{f}(w)$, it follows that $f(u)^{-1} \cdot \phi(a) \cdot f(w)$ is a segment of $\bar{f}(u)^{-1} \cdot \phi(a) \cdot \bar{f}(w)$. So $\left|\bar{f}(u)^{-1} \cdot \phi(a) \cdot \bar{f}(w)\right|>1$. As $\bar{f}$ is prefeasible this implies that $\phi(a)=\bar{f}(u) y \bar{f}(w)^{-1}$ for some $y$. Hence, since $f \leq \bar{f}$, $\phi(a)=f(u) y^{\prime} f(w)^{-1}$ for some $y^{\prime}$. So $f(u) \leq f(u) y^{\prime}=\phi(a) \cdot f(w)$, contradicting our assumption.

\section{A subroutine finding $\bar{f}$}

Let input $D=(V, A), r, \phi$ for the cohomology feasibility problem (6) be given. We may assume that for any arc $a=(u, w), a^{-1}=(w, u)$ is also an $\operatorname{arc}$ of $D$, with $\phi\left(a^{-1}\right)=\phi(a)^{-1}$. Let moreover $f: V \longrightarrow B_{k}$ be given.

If $f$ is pre-feasible output $\bar{f}:=f$. If $f$ violates (9) output $\bar{f}:=\infty$. If none of these applies, perform the following iteration:

Iteration: Choose an $\operatorname{arc} a=(u, w)$ satisfying $\left|f(u)^{-1} \cdot \phi(a) \cdot f(w)\right|>1$ and $f(w) \leq \underline{\phi}(a)^{-1} \cdot f(u)$. (Such an arc exists by (4). As (9)(iii) is not violated, we know $f(u) \leq \phi(a) \cdot f(w)$.)

Let $x$ be obtained from $\phi(a) \cdot f(w)$ by deleting the last symbol; reset $f(u):=x$,

and iterate.

Proposition 3. At each iteration, $\sum_{v}|f(v)|$ strictly increases. 
Proof. Since $f(u) \leq \phi(a) \cdot f(w)$ and $\left|f(u)^{-1} \cdot \phi(a) \cdot f(w)\right|>1, x$ is strictly larger than the original $f(u)$.

This directly implies:

Proposition 4. After at most $(\sigma+1)|V|^{2}$ iterations the subroutine stops.

Proof. After $(\sigma+1)|V|^{2}$ iterations, by Proposition 3 there exists a vertex $u$ such that $|f(u)| \geq(\sigma+1)|V|$. Then (9)(ii) is violated.

Moreover we have:

Proposition 5. In the iteration, resetting $f$ does not change $\bar{f}$.

Proof. We must show that $x \leq \bar{f}(u)$ if $\bar{f}$ is finite. If there exists $y$ such that $\phi(a)=\bar{f}(u) y \bar{f}(w)^{-1}$ then

$$
f(w) \leq \bar{f}(w) \leq \bar{f}(w) y^{-1}=\phi(a)^{-1} \cdot \bar{f}(u) \leq \phi(a)^{-1} \cdot f(u)
$$

(since $f(u) \leq \bar{f}(u) \leq \phi(a))$. This contradicts the choice of $a$ in the iterations. Therefore, since $\bar{f}$ is pre-feasible, we know $\left|\bar{f}(u)^{-1} \cdot \phi(a) \cdot \bar{f}(w)\right| \leq 1$.

Since $f(w) \not \leq \phi\left(a^{-1}\right) \cdot f(u)$, by Lemma 2 the last symbol of $f(u)^{-1} \cdot \phi(a) \cdot f(w)$ is equal to the last symbol of $f(w)$. Hence (since $f(w) \leq \bar{f}(w)) f(u)^{-1} \cdot \phi(a) \cdot f(w) \leq$ $f(u)^{-1} \cdot \phi(a) \cdot \bar{f}(w)$. Since $f(u) \leq \phi(a) \cdot f(w)$ it follows that $\phi(a) \cdot f(w) \leq$ $\phi(a) \cdot \bar{f}(w)$. Let $y$ be obtained from $\phi(a) \cdot \bar{f}(w)$ by deleting the last symbol. Then $x \leq y \leq \bar{f}(u)$, since $\left|\bar{f}(u)^{-1} \cdot \phi(a) \cdot \bar{f}(w)\right| \leq 1$.

\section{Algorithm for the cohomology feasibility problem}

Let input $D=(V, A), r, \phi$ for the cohomology feasibility problem (6) be given. Again we may assume that for each $\operatorname{arc} a=(u, w), a^{-1}=(w, u)$ is also an arc, with $\phi\left(a^{-1}\right)=\phi(a)^{-1}$. We find a feasible function $f$ (if there is one) as follows.

Let $W$ be the set of pairs $(v, x)$ with $v \in V$ and $x \in B_{k}$ such that there exists an arc $a=(v, w)$ with $1 \neq x \leq \phi(a)$. For every $(v, x) \in W$ let $f_{v, x}$ be the function defined by: $f_{v, x}(v):=x$ and $f_{v, x}\left(v^{\prime}\right):=1$ for each $v^{\prime} \neq v$. Let $E$ be the set of pairs $\left\{(v, x),\left(v^{\prime}, x^{\prime}\right)\right\}$ from $W$ for which $\bar{f}_{v, x} \vee \bar{f}_{v^{\prime}, x^{\prime}}$ is finite and pre-feasible. Let $E^{\prime}$ be the set of pairs $\{(u, x),(w, z)\}$ from $W$ for which there is an arc $a=(u, w)$ with $\phi(a)=x z^{-1}$. We search for a subset $X$ of $W$ such that each pair in $X$ belongs to $E$ and such that $X$ intersects each pair in $E^{\prime}$. This is a special case of the 2-satisfiability problem, and hence can be solved in polynomial time.

Proposition 6. If $X$ exists then the function $f:=\bigvee_{(v, x) \in X} \bar{f}_{v, x}$ is feasible. If $X$ does not exist then there is no feasible function.

Proof. First assume $X$ exists. Since $\bar{f}_{v, x} \vee \bar{f}_{v^{\prime}, x^{\prime}}$ is finite and pre-feasible for each two $(v, x),\left(v^{\prime}, x^{\prime}\right)$ in $X, f$ is finite and $f(r)=1$. Moreover, suppose $\mid f(u)^{-1} \cdot \phi(a)$. 
$f(w) \mid>1$ for some $\operatorname{arc} a=(u, w)$. By definition of $f$ there are $(v, x),\left(v^{\prime}, x^{\prime}\right) \in X$ such that $f(u)=\bar{f}_{v, x}(u)$ and $f(w)=\bar{f}_{v^{\prime}, x^{\prime}}(w)$ for $(v, x),\left(v^{\prime}, x^{\prime}\right) \in X$. As $\bar{f}_{v, x} \vee \bar{f}_{v^{\prime}, x^{\prime}}$ is pre-feasible, $\phi(a)=\bar{f}_{v, x}(u) y \bar{f}_{v^{\prime}, x^{\prime}}(w)^{-1}$ for some $y$. Then $|y|>1$. Split $y=b c^{-1}$ with $b$ and $c$ nonempty. Then $(u, f(u) b) \in X$ or $(w, f(w) c) \in X$ since $X$ intersects each pair in $E^{\prime}$. If $(u, f(u) b) \in X$ then $f(u) b=f_{u, f(u) b}(u) \leq$ $\bar{f}_{u, f(u) b}(u) \leq f(u)$, a contradiction. If $(w, f(w) c) \in X$ one obtains similarly a contradiction.

Assume conversely that there exists a feasible function $f$. Let $X$ be the set of pairs $(v, x) \in X$ with the property that $x \leq f(v)$. Then $X$ intersects each pair in $E^{\prime}$. For suppose that for some arc $a=(u, w)$ with $\phi(a)=x z^{-1}$ and $x \neq 1 \neq z$, one has $(u, x) \notin X$ and $(w, z) \notin X$, that is, $x \leq f(u)$ and $z \leq f f(w)$. This however implies $\left|f(u)^{-1} \cdot \phi(a) \cdot f(w)\right| \geq 2$, a contradiction.

Moreover, each pair in $X$ belongs to $E$. For let $(v, x),\left(v^{\prime}, x^{\prime}\right) \in X$. We show that $\left\{(v, x),\left(v^{\prime}, x^{\prime}\right)\right\} \in E$, that is, $f^{\prime}:=\bar{f}_{v, x} \vee \bar{f}_{v^{\prime}, x^{\prime}}$ is pre-feasible. As $\bar{f}_{v, x} \leq f$ and $\bar{f}_{v^{\prime}, x^{\prime}} \leq f, f^{\prime}$ is finite and $f^{\prime}(r)=1$. Consider an arc $a=(u, w)$ with $\left|f^{\prime}(u)^{-1} \cdot \phi(a) \cdot f^{\prime}(w)\right|>1$. We may assume $f^{\prime}(u)=\bar{f}_{v, x}(u)$ and $f^{\prime}(w)=\bar{f}_{v^{\prime}, x^{\prime}}(w)$ (since $\bar{f}_{v, x}$ and $\bar{f}_{v^{\prime}, x^{\prime}}$ themselves are pre-feasible). To show $\phi(a)=f^{\prime}(u) y f^{\prime}(w)^{-1}$ for some $y$, by (4) we may assume $f^{\prime}(w) \leq \phi\left(a^{-1}\right) \cdot f^{\prime}(u)$. So by Lemma 2 , the last symbol of $f^{\prime}(u)^{-1} \cdot \phi(a) \cdot f^{\prime}(w)$ is equal to the last symbol of $f^{\prime}(w)$.

Suppose now that $f^{\prime}(u) \not \subset(a) \cdot f^{\prime}(w)$. Then by Lemma 2 , the first symbol of $f^{\prime}(u)^{-1} \cdot \phi(a) \cdot f^{\prime}(w)$ is equal to the first symbol of $f^{\prime}(u)^{-1}$. Since $f^{\prime} \leq f$ this implies that $f^{\prime}(u)^{-1} \cdot \phi(a) \cdot f^{\prime}(w)$ is a segment of $f(u)^{-1} \cdot \phi(a) \cdot f(w)$. This contradicts the fact that $\left|f(u)^{-1} \cdot \phi(a) \cdot f(w)\right| \leq 1$.

So $f^{\prime}(u) \leq \phi(a) \cdot f^{\prime}(w)$. As $f_{v^{\prime}, x^{\prime}}(u) \leq f^{\prime}(u)$ and $\left|f^{\prime}(u)^{-1} \cdot \phi(a) \cdot f^{\prime}(w)\right|>1$ it follows that $\left|\overline{\bar{f}}_{v^{\prime}, x^{\prime}}(u)^{-1} \cdot \phi(a) \cdot f^{\prime}(w)\right|>1$. As $f^{\prime}(w)=\bar{f}_{v^{\prime}, x^{\prime}}(w)$ we have $\mid \bar{f}_{v^{\prime}, x^{\prime}}(u)^{-1}$. $\phi(a) \cdot \bar{f}_{v^{\prime}, x^{\prime}}(w) \mid>1$. As $\bar{f}_{v^{\prime}, x^{\prime}}$ is pre-feasible, $\phi(a)=\bar{f}_{v^{\prime}, x^{\prime}}(u) y \bar{f}_{v^{\prime}, x^{\prime}}(w)^{-1}$ for some $y$. So $f^{\prime}(u) \leq \phi(a) \cdot f^{\prime}(w)=\bar{f}_{v^{\prime}, x^{\prime}}(u) y$. Hence $\bar{f}_{v^{\prime}, x^{\prime}}(u) y=f^{\prime}(u) y^{\prime}$ for some $y^{\prime}$. It follows that $\phi(a)=f^{\prime}(u) y^{\prime} f^{\prime}(w)^{-1}$.

Thus we have:

THEOREM 1. The cohomology feasibility problem for free boolean groups is solvable in time bounded by a polynomial in $|A|+\sigma+k$.

\section{Graphs on surfaces and homologous functions}

Let $G=(V, E)$ be an undirected graph embedded in a compact surface. For each edge $e$ of $G$ choose arbitrarily one of the faces incident with $e$ as the lefthand face of $e$, and the other as the right-hand face of $e$. (They might be one and the same face.) Let $\mathcal{F}$ denote the set of faces of $G$, and let $R$ be one of the faces of $G$. We call two functions $\phi, \psi: E \longrightarrow B_{k} R$-homologous if there exists 
a function $f: \mathcal{F} \longrightarrow B_{k}$ such that

(i) $f(R)=1$;

(ii) $f(F)^{-1} \cdot \phi(e) \cdot f\left(F^{\prime}\right)=\psi(e)$ for each edge $e$, where $F$ and $F^{\prime}$ are the left-hand and right-hand face of $e$ respectively.

The relation to cohomologous is direct by duality. The dual graph $G^{*}=$ $\left(\mathcal{F}, E^{*}\right)$ of $G$ has as vertex set the collection $\mathcal{F}$ of faces of $G$, while for any edge $e$ of $G$ there is an edge $e^{*}$ of $G^{*}$ connecting the two faces incident with $e$. Let $D^{*}$ be the directed graph obtained from $G^{*}$ by orienting each edge $e^{*}$ from the left-hand face of $e$ to the right-hand face of $e$. Define for any function $\phi$ on $E$ the function $\phi^{*}$ on $E^{*}$ by $\phi^{*}\left(e^{*}\right):=\phi(e)$ for each $e \in E$. Then $\phi$ and $\psi$ are $R$-homologous (in $G$ ), if and only if $\phi^{*}$ and $\psi^{*}$ are $R$-cohomologous (in $D^{*}$ ).

\section{Enumerating homology classes}

Let $G=(V, E)$ be an undirected graph embedded in a surface and let $r, s \in V$, such that no loop is attached at $r$ or $s$. We call a collection $\Pi=\left(P_{1}, \ldots, P_{k}\right)$ of $r-s$ walks an $r-s$ join (of size $k$ ) if:

(i) each $P_{i}$ traverses $r$ and $s$ only as first and last vertex respectively;

(ii) each edge is traversed at most once by the $P_{1}, \ldots, P_{k}$;

(iii) $P_{i}$ does not cross itself or any of the other $P_{j}$;

(iv) $P_{1}, \ldots, P_{k}$ occur in this order cyclically at $r$.

Note that any solution of (2) can be assumed to be an $r-s$ join.

For any $r-s$ join $\Pi=\left(P_{1}, \ldots, P_{k}\right)$ let $\phi_{\Pi}: E \longrightarrow B_{k}$ be defined by:

$$
\begin{array}{rll}
\phi_{\Pi}(e) & :=g_{i} & \text { if walk } P_{i} \text { traverses } e(i=1, \ldots, k) \\
& :=1 & \text { if } e \text { is not traversed by any of the } P_{i}
\end{array}
$$

Let $R$ be one of the faces of $G$. Note that if $\phi$ is $R$-homologous to $\phi_{\Pi}$ then for each vertex $v \neq r, s$ we have

$$
\phi\left(e_{1}\right)^{\varepsilon_{1}} \cdot \ldots \cdot \phi\left(e_{t}\right)^{\varepsilon_{t}}=1
$$

where $F_{0}, e_{1}, F_{1}, \ldots, F_{t-1}, e_{t}, F_{t}$ are the faces and edges incident with $v$ in cyclic order (with $F_{t}=F_{0}$ ), and where $\varepsilon_{j}:=+1$ if $F_{j-1}$ is the left-hand face of $e_{j}$ and $F_{j}$ is the right-hand face of $e_{j}$, and $\varepsilon_{j}:=-1$ if $F_{j-1}$ is the right-hand face of $e_{j}$ and $F_{j}$ is the left-hand face of $e_{j}$. (If $F_{j-1}=F_{j}$ we should be more careful.) This follows from the fact that (14) holds for $\phi=\phi_{\Pi}$ and that (14) is invariant for $R$-homologous functions. 
We now consider the following problem:

given: a connected undirected graph cellularly embedded on a surface $S$, vertices $r, s$ of $G$, such that $G-\{r, s\}$ is connected and $r$ and $s$ are not connected by an edge, a face $R$ of $G$, and a natural number $k$;

find: functions $\phi_{1}, \ldots, \phi_{N}: E \longrightarrow B_{k}$ such that for each $r-s$ join $\Pi$ of size $k, \phi_{\Pi}$ is $R$-homologous to at least one of $\phi_{1}, \ldots, \phi_{N}$.

(A graph is cellularly embedded if each face is homeomorphic with an open disk.)

THEOREM 2. For any fixed surface $S$, problem (15) is solvable in time bounded by a polynomial in $|V|+|E|$.

Proof. If $e$ is any edge connecting two different vertices $\neq r, s$, we can contract $e$. Any solution of (15) for the modified graph directly yields a solution for the original graph (by (14)). So we may assume $V=\{r, s, v\}$ for some vertex $v$. Similarly, we may assume that $G$ has no loops that bound an open disk.

Call two edges parallel if and only if they form the boundary of an open disk in $S$ not containing $R$. Let $p$ be the number of parallel classes and let $f^{\prime}$ denote the number of faces that are bounded by at least three edges. So $2 p \geq 3 f^{\prime}$. By Euler's formula, $4+f^{\prime} \geq p+\chi(S)$, where $\chi(S)$ denotes the Euler characteristic of $S$. This implies $12+2 p \geq 12+3 f^{\prime} \geq 3 p+3 \chi(S)$ and hence $p \leq 12-3 \chi(S)$. That is, for fixed $S, p$ is bounded.

Let $E^{\prime}$ be a subset of $E$ containing one edge from every parallel class. Note that any $B_{k}$-valued function on $E$ is $R$-homologous to a $B_{k}$-valued function that has value 1 on all edges not in $E^{\prime}$.

Let $\Pi=\left(P_{1}, \ldots, P_{k}\right)$ be an $r-s$ join such that no $P_{i}$ traverses two edges $e, e^{\prime}$ consecutively that are parallel. For any 'path' $e, v, e^{\prime}$ in $E^{\prime}$ of length two, with $e$ and $e^{\prime}$ incident with vertex $v$ and $e$ and $e^{\prime}$ not parallel, let $f\left(\Pi, e, v, e^{\prime}\right)$ be the number of times the $P_{i}$ contain $\tilde{e}, v, \tilde{e}^{\prime}$, for some $\tilde{e}$ parallel to $e$ and some $\tilde{e}^{\prime}$ parallel to $e^{\prime}$. (Here $e$ or $e^{\prime}$ is assumed to have an orientation if it is a loop.)

Now up to $R$-homology and up to a cyclic permutation of the indices of $P_{1}, \ldots, P_{k}, \Pi$ is fully determined by the numbers $f\left(\Pi, e, v, e^{\prime}\right)$. This follows directly from the fact that the $P_{i}$ do not have (self-)crossings.

So to enumerate $\phi_{1}, \ldots, \phi_{N}$ it suffices to choose for each path $e, v, e^{\prime}$ a number $g\left(e, v, e^{\prime}\right) \leq|E|$. Since $\left|E^{\prime}\right|=p \leq 9-3 \chi(S)$ there are at most $(|E|+1)^{(12-3 \chi(S))^{2}}$ such choices. For each choice we can find in polynomial time an $r-s$ join $\Pi$ with $f\left(\Pi, e, v, e^{\prime}\right)=g\left(e, v, e^{\prime}\right)$ for all $e, v, e^{\prime}$ if it exists. Enumerating the $\phi_{\Pi}$ gives the required enumeration.

\section{Induced circuits}

THEOREM 3. For each fixed surface $S$, there is a polynomial-time algorithm that gives for any graph $G=(V, E)$ embedded on $S$ and any two vertices $r, s$ of 
$G$ a maximum number of $r-s$ paths each two of which form an induced circuit.

Proof. It suffices to show that for each fixed natural number $k$ we can find in polynomial time $k r-s$ paths each two of which form an induced circuit, if they exist.

We may assume that $G-\{r, s\}$ is connected, that $r$ and $s$ are not connected by an edge, and that $G$ is cellularly embedded. Choose a face $R$ of $G$ arbitrarily. By Theorem 2 we can find in polynomial time a list of functions $\phi_{1}, \ldots, \phi_{N}$ : $A \longrightarrow B_{k}$ such that for each $r-s$ join $\Pi, \phi_{\Pi}$ is $R$-homologous to at least one of the $\phi_{j}$.

Consider the (directed) dual graph $D^{*}=\left(\mathcal{F}, A^{*}\right)$ of $G$ (see Section 6). We extend $D^{*}$ to a graph $D^{+}=\left(\mathcal{F}, A^{+}\right)$as follows.

For every pair of vertices $F, F^{\prime}$ of $D^{*}$ and every $F-F^{\prime}$ path $\pi$ (not necessarily directed) on the boundary of one face or of two adjacent faces of $D^{*}$, extend the graph with an $\operatorname{arc} a_{\pi}$ from $F$ to $F^{\prime}$. (Note that there are only a polynomially bounded number of such paths.) For each $\phi: A \longrightarrow B_{k}$ define $\phi^{+}: A^{+} \longrightarrow B_{k}$ by $\phi^{+}\left(e^{*}\right):=\phi(e)$ and

$$
\phi^{+}\left(a_{\pi}\right):=\phi\left(e_{1}\right)^{\varepsilon_{1}} \cdot \ldots \cdot \phi\left(e_{t}\right)^{\varepsilon_{t}}
$$

for any path $\pi=\left(e_{1}^{*}\right)^{\varepsilon_{1}} \ldots\left(e_{t}^{*}\right)^{\varepsilon_{t}}$. (Here $\varepsilon_{1}, \ldots, \varepsilon_{t} \in\{+1,-1\}$.)

By Theorem 1 we can find, for each $j=1, \ldots, N$ in polynomial time a function $\vartheta$ satisfying

$$
\begin{aligned}
& \text { (i) } \vartheta \text { is } R \text {-cohomologous to } \phi_{j}^{+} \text {in } D^{+} \text {, and } \\
& \text { (ii) }|\vartheta(b)| \leq 1 \text { for each } \operatorname{arc} b \text { of } D^{+} \text {, }
\end{aligned}
$$

provided that such a $\vartheta$ exists.

If we find a function $\vartheta$, for $i=1, \ldots, k$ let $Q_{i}$ be a shortest $r-s$ path traversing only the set of edges $e$ of $G$ with $\vartheta\left(e^{*}\right)=g_{i}$. If such paths $Q_{1}, \ldots, Q_{k}$ exist, and any two of them form an induced circuit, we are done (for the current value of $k)$.

We claim that, doing this for all $\phi_{1}, \ldots, \phi_{N}$, we find paths as required, if they exist. For let $\Pi:=\left(P_{1}, \ldots, P_{k}\right)$ form a collection of $k r-s$ paths any two of which form an induced circuit. Since $\Pi$ is an $r-s$ join, there exists a $j \in\{1, \ldots, N\}$ such that $\phi_{\Pi}$ and $\phi_{j}$ are $R$-homologous.

We first show that there exists a function $\vartheta$ satisfying (17), viz. $\vartheta:=\phi_{\Pi}^{+}$. To see this, we first show that $\phi_{\Pi}^{+}$is $R$-cohomologous to $\phi_{j}^{+}$in $D^{+}$. Indeed, $\phi_{\Pi}$ and $\phi_{j}$ are $R$-homologous in $G$. Hence there exists a function $f: \mathcal{F} \longrightarrow B_{k}$ such that $f(R)=1$ and such that

$$
f(F)^{-1} \cdot \phi_{\Pi}(e) \cdot f\left(F^{\prime}\right)=\phi_{j}(e)
$$

for each edge $e$, where $F$ and $F^{\prime}$ are the left-hand and right-hand face of $e$ respectively. This implies:

$$
f(F)^{-1} \cdot \phi_{\Pi}^{+}\left(e^{*}\right) \cdot f\left(F^{\prime}\right)=\phi_{j}^{+}\left(e^{*}\right) .
$$


Moreover, for every pair of vertices $F_{0}, F_{t}$ of $D^{*}$ and every $F_{0}-F_{t}$ path $\pi=$ $\left(e_{1}^{*}\right)^{\varepsilon_{1}} \ldots\left(e_{t}^{*}\right)^{\varepsilon_{t}}$ in $D^{*}$ on the boundary of at most two faces of $D^{*}$ we have (assuming $\left(e_{i}^{*}\right)^{\varepsilon_{i}}$ runs from $F_{i-1}$ to $F_{i}$ for $i=1, \ldots, t$ ):

$$
\begin{aligned}
& f\left(F_{0}\right)^{-1} \cdot \phi_{\Pi}^{+}\left(a_{\pi}\right) \cdot f\left(F_{t}\right) \\
& =\left(f\left(F_{0}\right)^{-1} \cdot \phi_{\Pi}\left(e_{1}\right)^{\varepsilon_{1}} f\left(F_{1}\right)\right) \cdot\left(f\left(F_{1}\right)^{-1} \cdot \phi_{\Pi}\left(e_{2}\right)^{\varepsilon_{2}} f\left(F_{2}\right)\right) . \\
& \ldots \cdot\left(f\left(F_{t-1}\right)^{-1} \cdot \phi_{\Pi}\left(e_{t}\right)^{\varepsilon_{1}} f\left(F_{t}\right)\right) \\
& =\phi_{j}\left(e_{1}\right)^{\varepsilon_{1}} \cdot \phi_{j}\left(e_{2}\right)^{\varepsilon_{2}} \cdot \ldots \cdot \phi_{j}\left(e_{t}\right)^{\varepsilon_{t}}=\phi_{j}^{+}\left(a_{\pi}\right) .
\end{aligned}
$$

So $\phi_{\Pi}^{+}$and $\phi_{j}^{+}$are $R$-cohomologous.

Next we show that $\left|\phi_{\Pi}^{+}(b)\right| \leq 1$ for each arc $b$ of $D^{+}$. Indeed, for any edge $e$ of $G$ we have $\phi_{\Pi}^{+}\left(e^{*}\right)=\phi_{\Pi}(e) \in\left\{1, g_{1}, \ldots, g_{k}\right\}$. So $\left|\phi_{\Pi}^{+}\left(e^{*}\right)\right| \leq 1$. Moreover, for any path $\pi=\left(e_{1}\right)^{\varepsilon_{1}}\left(e_{2}\right)^{\varepsilon_{2}} \ldots\left(e_{t}\right)^{\varepsilon_{t}}$ as above, $\phi_{\Pi}^{+}\left(a_{\pi}\right)=\phi_{\Pi}\left(e_{1}\right)^{\varepsilon_{1}} \ldots \ldots \cdot \phi_{\Pi}\left(e_{t}\right)^{\varepsilon_{t}}$. Since there exist two vertices $v^{\prime}, v^{\prime \prime}$ of $G$ such that each of $e_{1}, \ldots, e_{t}$ is incident with at least one of $v^{\prime}, v^{\prime \prime}$, we know that there exists at most one $i \in\{1, \ldots, k\}$ such that $P_{i}$ traverses at least one of the edges $e_{1}, \ldots, e_{t}$. Hence there is at most one generator occurring in $\phi_{\Pi}\left(e_{1}\right)^{\varepsilon_{1}} \ldots \ldots \phi_{\Pi}\left(e_{t}\right)^{\varepsilon_{t}}$. That is, $\left|\phi_{\Pi}^{+}\left(a_{\pi}\right)\right| \leq 1$. This shows that $\vartheta:=\phi_{\Pi}^{+}$satisfies (17).

Conversely, we must show that if $\vartheta$ satisfies (17), then $\vartheta$ gives paths $Q_{1}, \ldots, Q_{k}$ as above. Indeed, since $\vartheta$ is $R$-cohomologous to $\phi_{\Pi}^{+}$, for each $i=1, \ldots, k$, the set of edges $e$ of $G$ with $\vartheta\left(e^{*}\right)=g_{i}$ contains an $r-s$ path (since $\zeta:=\phi_{\Pi}^{+}$has the property that the subgraph $\left(V,\left\{e \in E \mid \zeta\left(e^{*}\right)\right.\right.$ contains the symbol $g_{i}$ an odd number of times $\}$ ) of $G$ has even degree at each vertex except at $r$ and $s$, and since this property is maintained under $R$-cohomology). Choose for each $i$ such a path $Q_{i}$. Suppose that, for some $i \neq j$, there exists an edge $e=\left\{v, v^{\prime}\right\}$ with $Q_{i}$ traversing $v$ and $Q_{j}$ traversing $v^{\prime}\left(v, v^{\prime} \notin\{r, s\}\right)$. Then there exist faces $F_{0}$ and $F_{t}$ of $G$ and an $F_{0}-F_{t}$ path $\pi=\left(e_{1}\right)^{\varepsilon_{1}} \ldots\left(e_{t}\right)^{\varepsilon_{t}}$ in $D^{*}$ on the boundary of the faces $v$ and $v^{\prime}$ of $D^{*}$ such that $\vartheta\left(e_{1}^{*}\right)^{\varepsilon_{1}} \cdot \ldots \vartheta\left(e_{t}^{*}\right)^{\varepsilon_{t}}$ contains both symbol $g_{i}$ and symbol $g_{j}$. Now

$$
\vartheta\left(a_{\pi}\right)=\vartheta\left(e_{1}^{*}\right)^{\varepsilon_{1}} \cdot \ldots \cdot \vartheta\left(e_{t}^{*}\right)^{\varepsilon_{t}}
$$

since this equation is invariant under $R$-cohomology and since it holds when $\vartheta$ is replaced by $\phi_{\Pi}^{+}$. So $\vartheta\left(a_{\Pi}\right)$ contains both symbol $g_{i}$ and $g_{j}$. This contradicts the fact that $\left|\vartheta\left(a_{\pi}\right)\right| \leq 1$.

So there is no edge connecting internal vertices of $Q_{i}$ and $Q_{j}$. Replacing each $Q_{i}$ by a chordless path $Q_{i}^{\prime}$ in $G$ that uses only vertices traversed by $Q_{i}$, we obtain paths as required.

We refer to [4] for an extension of the methods described above.

Acknowledgement. I am grateful to Paul Seymour for very carefully reading preliminary versions of this paper and for giving several helpful suggestions. 


\section{REFERENCES}

1. D. Bienstock, private communication, 1989.

2. C. McDiarmid, B. Reed, A. Schrijver, and B. Shepherd, Induced circuits in planar graphs, Report BS-R9106, CWI, Amsterdam, 1991.

3. A. Schrijver, Finding $k$ disjoint paths in directed planar graphs, Report BS-R9206, CWI, Amsterdam, 1992.

4. A. Schrijver, Disjoint paths in graphs on surfaces and combinatorial group theory, preprint, 1991.

CWI, Kruislaan 413, 1098 SJ Amsterdam, The Netherlands,

AND

Department of Mathematics, University of Amsterdam, Plantage MuiderGRACHT 24, 1018 TV Amsterdam, THE NEThERLANDS.

E-mail address: lex@cwi.nl 